\title{
PRMT5 suppresses DR4-mediated CCL20 release via NF-кB pathway
}

\author{
WANG DongSheng ${ }^{1,2}$, LIU Dan $^{1}$, GAO Jing ${ }^{1}$, LIU Min ${ }^{1}$, LIU ShiLian ${ }^{1}$, LIU YanXin ${ }^{1 *} \&$ \\ ZHENG DeXian ${ }^{1 *}$ \\ ${ }^{1}$ National Laboratory of Medical Molecular Biology, Institute of Basic Medical Sciences, Chinese Academy of Medical Sciences \& Peking Union \\ Medical College, Beijing 100005, China; \\ ${ }^{2}$ Department of Laboratory Medicine, Affiliated Hospital of North Sichuan Medical College, Nanchong 637000, China
}

Received December 27, 2011; accepted February 8, 2012

\begin{abstract}
Construct expression vectors of pCMV-DR4-HA and pCMV-PRMT5-Flag, and transfect them into HEK293 cells to identify the interaction between TRAIL-R1 and PRMT5 and the molecular mechanism underlying DR4-mediated inhibition of chemokine CCL20 release via TRAIL receptor 1 (DR4). Inflammatory cytokine was detected by RT-PCR and ELISA after TRAIL-R1 and/or PRMT5 transfection, respectively. NF- $\mathrm{kB}$ activity was detected by Dual Luciferase Reporter Gene Assay. ERK1/2 phosphorylation was analyzed by Western blot. PRMT5 could inhibit DR4-activated NF- $\kappa$ B activity and ERK1/2 phosphorylation. PRMT5 could inhibit NF- $\mathrm{KB}$ activition, ERK1/2 phosphorylation as well as CCL20 secretion via binding with DR4 in HEK293 cell, suggesting that PRMT5 may involve in DR4 dependent immune regulation.
\end{abstract}

TRAIL, PRMT5, cytokine, CCL20

Citation: Wang D S, Liu D, Gao J, et al. PRMT5 suppresses DR4-mediated CCL20 release via NF-кB pathway. Chin Sci Bull, 2012, 57: 4351-4355, doi: 10.1007/s11434-012-5511-6

Tumor necrosis factor (TNF)-related apoptosis-inducing ligand (TRAIL) is a member of the TNF superfamily [1]. Intensive researches have demonstrated that both membrane-bound and soluble-form TRAIL rapidly induce apoptosis of various tumor cells with the expression of TRAIL receptor 1 (TRAIL-R1) or 2 (TRAIL-R2), which are also known as death receptor 4 (DR4) or 5 (DR5). As a matter of fact, TRAIL is able to bind with its five cognate receptors in human being: two death receptors mediating cell death by apoptosis, DR4/TRAIL-R1 and DR5/TRAIL-R2/killer; three decoy receptors, DcR1/TRAIL-R3/TRID, DcR2/ TRAIL-R4/TRUNDD, and secreted osteoprotegerin (OPG), which could bind with TRAIL, but could not mediate apoptotic signaling. TRAIL induces apoptosis upon binding with death receptor in various tumor cells, but not in most normal cells [2,3]. Overexpression of death receptor in certain normal cell lines can also trigger apoptosis [4]. Once bind-

*Corresponding authors (email: zhengdx@pumc.edu.cn; liuyx2000@tom.com) ing with TRAIL, the intracellular domain of death receptor recruits a series of adapter molecules forming death-inducing signaling complex (DISC). Then the classical apoptotic pathway is activated and then results in cell death. However, recent report [5] showed that other signaling pathway can be also activated by the molecules in DISC complex.

It is shown [6] that PRMT5, an arginine methylase, can bind with DR4 and then inhibit the activation of NF- $\mathrm{kB}$, which is not dependent on the methylation activity of PRMT5. PRMT5 results in protein methylation after translation through the regulation of certain biological gene transcription, RNA process. Therefore, PRMT5 plays an important role in regulating cell proliferation. PRMT5 can also affect the release of cytokines through epigenetic regulation [7].

Recently, a growing number of studies demonstrate that TRAIL not only induces apoptosis, but also activates nonapoptotic signaling pathways, such as MAPK, AKT, PKC, NF- $\kappa B$ and etc. $[8,9]$. There are some reports indicating that 
TRAIL induces IL- 8 and MCP-1 expression in certain cell lines. Our previous study shows that TRAIL promotes the release of CCL20 through activating the NF- $\kappa B$ pathway $[10,11]$. Overexpression of DR4 can also cause the secretion of CCL20 by similar mechanism. However, the specific signaling molecule has not been elucidated. In the present study, we explored DR4 interacting proteins and the molecular mechanism regulating CCL20 release and found that DR4 associated with PRMT5 directly and regulated CCL20 release via NF- $\kappa$ B pathway.

\section{Materials and methods}

\subsection{Cell culture}

HEK293T cell line (human embryonic kidney cell) used in the study was purchased from the Cell Center, Institute of Basic Medical Science, Chinese Academy of Medical Science. The cells were cultured in DMEM (Gibco ${ }^{\circledR}$, USA) supplemented with $10 \%$ fetal bovine serum (FBS, Gibco ${ }^{\circledR}$ ) at $37^{\circ} \mathrm{C}$ in an incubator with controlled humidified atmosphere containing $5 \% \mathrm{CO}_{2}$.

\subsection{Plasmids and reagents}

Plasmid pCMV-PRMT5 was kindly provided by Professor BaoShi Lai (Institute of Genetics and Developmental Biology, Chinese Academy of Sciences, Beijing). The DR4 expressing vector pCDNA3.1-DR4 and the NF- $\kappa \mathrm{B}$ reporter plasmid pGL3-NF- $\kappa \mathrm{B}$ were prepared previously in our lab. Restriction enzymes, T4 ligase, pMD18-T vector and DNA molecular weight marker were purchased from TaKaRa Co. Plasmid DNA extraction kit, PCR product purification kit, and gel DNA extraction kit were purchased from Tiangen Biological Technology Co., Ltd. (Beijing, China). Fetal bovine serum and DMEM medium (high glucose) were purchased from Gibco $^{\circledR}$. Transfection reagent Lipofectamine $^{\mathrm{TM}} 2000$ was purchased from Invitrogen ${ }^{\mathrm{TM}}$ (USA). Common protein lysis buffer or protein extracting reagents were purchased from Gailing Co., Ltd. (Beijing, China). PVDF membrane was purchased from Millipore Corporation (USA). Standard molecular weight marker of pre- stained protein was purchased from Saibeisheng Co., Ltd (Beijing, China). Chemical luminescence kit was bought from General Electric Company (GE, USA). Monoclonal antibodies against phosphorylated ERK1/2 and GAPDH were purchased from Cell Signaling Technology, Inc. (USA).

\subsection{Construction of the recombinant plasmid}

Full-length DR4 cDNA was amplified by PCR using pCDNA3.1-DR4 plasmid DNA as a template, 5'-GGAATTCGGATGGCGCCACCACCAGCTAG-3' and 5'-CGCTCGAGCTCACTTGTCATCGTCGTCCTTGTAGTCCTCC-
AAGGACACGGCAG-3' as upstream and downstream primers. EcoRI and XhoI restriction sites were introduced in the upstream primer 5' end and downstream primer 5' end, respectively. PCR reaction was set up as follows: $5 \times$ buffer $4 \mu \mathrm{L}$, dNTP $2.5 \mu \mathrm{mol}$, each primer $10 \mathrm{pmol}$, primer star enzyme $0.6 \mathrm{U}$, template DNA $100 \mathrm{ng}$, double distilled water to $20 \mu \mathrm{L}$ of total reaction volume. PCR condition was as follows: $98^{\circ} \mathrm{C}$ for $6 \mathrm{~min}, 98^{\circ} \mathrm{C}$ for $15 \mathrm{~s}, 58^{\circ} \mathrm{C}$ for $40 \mathrm{~s}, 72^{\circ} \mathrm{C}$ for $2 \mathrm{~min}, 35$ cycles, then $72^{\circ} \mathrm{C}$ terminal extension for 10 min. PCR product was analyzed by agarose gel electrophoresis to confirm the correct length of DR4 (1584 bp). The PCR product was digested by EcoRI and XhoI, respectively. The digestion reaction was as follows: PCR product $1 \mu \mathrm{g}$, EcoRI $10 \mathrm{U}$, XhoI $10 \mathrm{U}, \mathrm{H}$ buffer $1 \mu \mathrm{L}$, double distilled water to $20 \mu \mathrm{L}$ of reaction volume. Gel recovered DR4 was ligated with pCMV-HA plasmid predigested with the same restriction enzymes with a concentration ratio of $1: 5$. The ligated products were transformed into DH5 $\alpha$ competent cells. The cells were cultured in LB plate containing 50 $\mu \mathrm{g} / \mathrm{mL}$ ampicilin at $37^{\circ} \mathrm{C}$ for $24 \mathrm{~h}$. Mono-colony was picked and the plasmid DNA was extracted and identified by restriction enzyme digestion and sequencing. The correct construct was named as pCMV-HA-DR4 for subsequent experiments.

\subsection{Co-immunoprecipitation}

PCMV-Flag-PRMT5 and pCMV-HA-DR4 plasmids were co-transfected into HEK293 cells. The cells were collected and lysed with $500 \mu \mathrm{L}$ common protein lysis buffer containing protease inhibitors on ice for $30 \mathrm{~min}$. The cell lysate was collected by centrifugation at $12000 \times g$ and $4^{\circ} \mathrm{C}$ for 10 $\min$. The $2 \mu \mathrm{g}$ of rabbit anti-HA polyclonal antibody was added into the lysate and oscillated at $4^{\circ} \mathrm{C}$ overnight and then $30 \mu \mathrm{L}$ protein $\mathrm{A}$ agarose bead suspension was added and oscillated at $4^{\circ} \mathrm{C}$ for $6 \mathrm{~h}$. Agarose beads were collected by centrifugation at $2500 \times g$ for $5 \mathrm{~min}$ and washed 3 times with protein lysis buffer, and then boiled for $5 \mathrm{~min}$ in $20 \mu \mathrm{L}$ protein sample buffer followed by SDS-PAGE. The target band was detected by Western blot assay using anti-HA monoclonal antibody.

\subsection{Western blot assay}

Cells were harvested and washed twice with cold PBS, and then lysed in $50 \mu \mathrm{L}$ ice-cold lysis buffer for $30 \mathrm{~min}$. Protein samples were boiled for $10 \mathrm{~min}$ in sample buffer and subjected to SDS-PAGE. The proteins in the gel were transferred on polyvinylidene difluoride membrane (PVDF). The membrane was blocked with skimmed milk at room temperature for $1 \mathrm{~h}$ and then incubated in the first antibody dilution at $4{ }^{\circ} \mathrm{C}$ overnight, and then washed 3 times with TBST buffer for $10 \mathrm{~min}$. The membrane was then incubated with the peroxidase labeled secondary antibody and washed with TBST buffer. The interested protein band was visual- 
ized by using ECL system and exposed to Kodak X-omat BT film [12].

\subsection{Dual-luciferase reporter assay}

The dual-luciferase reporter assay was carried out according to the manufactural instruction of Promega Corporation (USA). Briefly, 293T cells were co-transfected by reporter and internal control plasmids in a ratio of $50: 1$. After $48 \mathrm{~h}$ cultivation, cells were collected and lysed. The lysate was added into the wells $(100 \mu \mathrm{L} /$ well $)$ of 24 -well plate and mildly oscillated for $15 \mathrm{~min}$. The samples were placed at room temperature before measuring and $25 \mu \mathrm{L}$ LARII was added to each well. Set delay for $2 \mathrm{~s}$, measure for 10 s. 25 $\mu \mathrm{L}$ Stop\&Glo reagent was continually added and mixed. Fluorescence of the internal control Renilla reniformis luciferase was measured. Firefly luciferase activity was standardized by the sample with the Renilla reniformis luciferase activity. Then the relative luciferase activity was calculated.

\subsection{Inflammatory cytokine detection by RT-PCR and ELISA}

Cells were co-transfected according to the manufatural instruction of Lipofectamine ${ }^{\mathrm{TM}} 2000$ specification with: (1) pCMV-Flag $(2 \mu \mathrm{g})$ and pCMV-HA $(2 \mu \mathrm{g})$; (2) pCDNA3.0DR4 $(2 \mu \mathrm{g})$ and pCMV-Flag $(2 \mu \mathrm{g}) ;(3)$ pCMV-Flag $(2 \mu \mathrm{g})$ and pCMV-HA-DR4 ( $2 \mu \mathrm{g})$; (4) pCMV-Flag-PRMT5 $(2 \mu \mathrm{g})$ and pCMV-HA $(2 \mu \mathrm{g})$; (5) pCMV-Flag-PRMT5 $(2 \mu \mathrm{g})$ and pCMV-HA-DR4 $(2 \mu \mathrm{g})$. RNA extraction and cDNA synthesis were referred to the experimental guidance of Promega Corporation. PCR primers for each cytokines were as follows: IL8, 5'-ACTGAGAGTGATTGAGAGTGGACC-3', 5'-AACCCTCTGCACCCAGTTTTC-3'; TNF- $\alpha, 5^{\prime}$-CCAGGGACCTCTCTCTAATCAGC-3'， 5'-CTCAGCTTGAGGGTTTGCTACA-3'; CCL20, 5'-AGAGTTTGCTCCTGGCTG-3', 5'-GGATGAAGAATACGGTCTGTG-3'; MIP-1 $\beta$, 5'-GGAAGCTTCCTCGCAACTTTG-3', 5'-GCTCAGGTGACCTTCCCTGAA-3'; GAPDH, 5'-CCGTCTAGAAAAACCTGCC-3', 5'-AGCCAAATTCGTTGTCATACC-3'. All PCRs were run for 40 cycles. The PCR for internal control GAPDH was run for 30 cycles. ELISA was carried out according to the manufactural instruction (R\&D Corporation, USA).

\section{Results}

\subsection{PRMT5 suppresses mRNA expression of cytokine}

First all, we checked the influence of DR4 and/or PRMT5 over-expression in inflammatory cytokine expression. HEK293T cells were transfected with pCMV-Flag-PRMT5 and/or pCMV-HA-DR4 or pCDNA3.1-DR4. Inflammatory cytokine mRNA expression was detected by RT-PCR. As shown in Figure 1, the expressions of CCL20, IL-8, TNF $\alpha$, $M I P-1 \alpha$, and $M I P-1 \beta$ were significantly increased in DR4 over-expression cells, but suppressed markedly in PRMT5 and DR4 over-expressed cells. However, there was no change in the cytokine expression observed in PRMT5overexpressed cells, suggesting that PRMT5 suppresses the mRNA expression of the inflammatory cytokine.

\subsection{DR4 binds with PRMT5}

Next we investigated whether DR4 interacts with PRMT5 by co-immunoprecipitation followed by Western blot assay. As shown in Figure 2, anti-DR4 antibody immunoprecipitation could well pull down PRMT5 in pCMV-HA-DR4 and pCMV-Flag-PRMT5 co-transfected cells, suggesting that DR4 binds with PRMT5, which provides a molecular mechanism that PRMT5 suppresses the inflammatory cytokine expression.

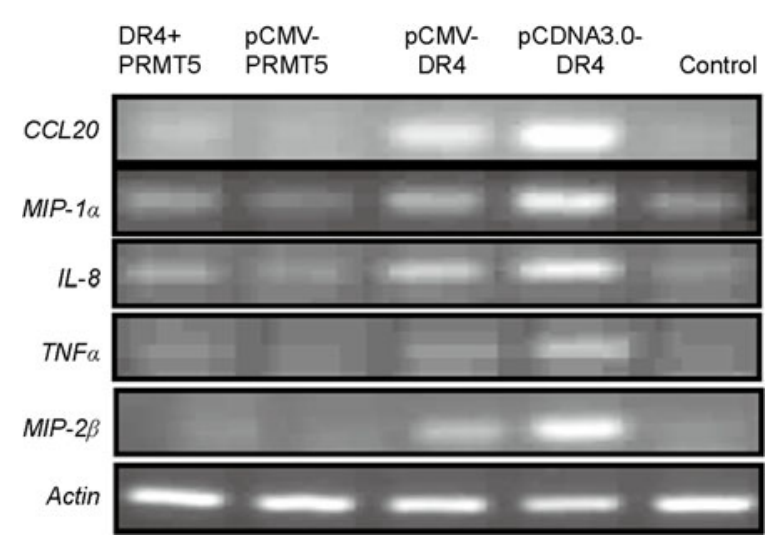

Figure 1 The mRNA expression of inflammatory cytokine in the HEK293T cells with DR4 and/or PRMT5 over-expression. HEK293T cells were transfected with pCMV-HA-DR4 or pCDNA3.0-DR4 and/or pCMV-Flag-PRMT5, respectively. The mRNA expression was detected by RT-PCR.

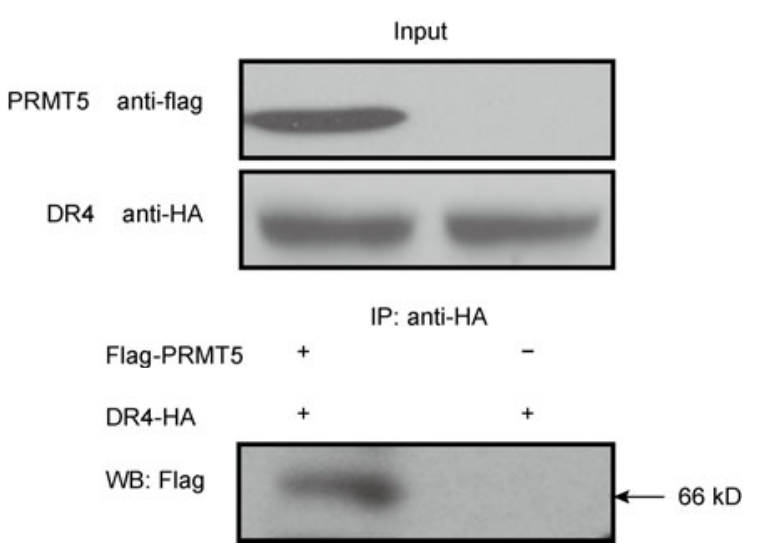

Figure 2 DR4 binds with PRMT5 in HEK293T cells identified by coimmunoprecipitation and Western blot assay. HEK293T cells were transfected with pCMV-HA-DR4 (DR4) and/or pCMV-Flag-PRMT5 (PRMT5). The cell lysates were subjected to co-immunoprecipitation and Western blot assay. 


\subsection{PRMT5 suppresses CCL20 protein expression}

Detection of CCL20 protein expression by ELISA showed that CCL20 protein expression was significantly increased in the cells with DR4 over-expression, while decreased in the cells with DR4 plus PRMT5 over-expression, but made no change in the cells with PRMT5 over-expression (Figure 3), suggesting that PRMT5 inhibits CCL20 expression.

\subsection{PRMT5 inhibits NF-kB activity}

We further analyzed NF-kB activity by Dual-luciferase reporter gene assay. As shown in Figure 4, NF- $\mathrm{kB}$ activity was activated in DR4 over-expressed cells, which was consistent with our previous report [11]. However, the activity of NF- $\mathrm{KB}$ was inhibited in PRMT5 over-expressed cells. These data provide an evidence that PRMT5 inhibits CCL20 expression via inhibiting NF- $\mathrm{KB}$ activation.

\subsection{PRMT5 blocks ERK1/2 phosphorylation}

Finally, we further tested phosphorylation of ERK1/2 in the cells with DR4 plus PRMT5 over-expression by Western blot assay. As shown in Figure 5, phosphorylation of ERK1/2 was increased in the cells with DR4 over-expression, but blocked markedly in the cells with PRMT5 over-expression.

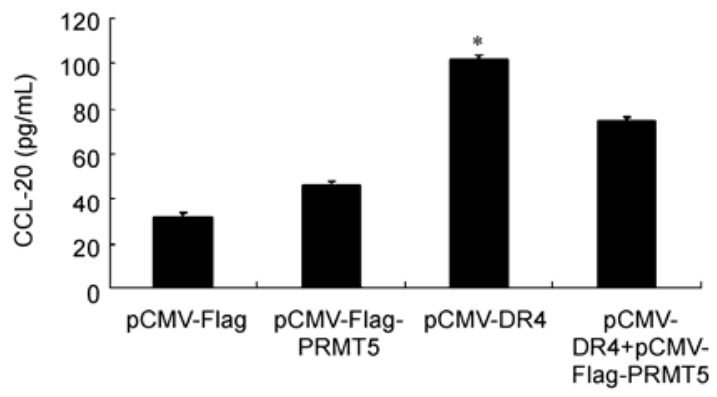

Figure 3 CCL20 expression in HEK293T cells with over-expression of DR4 and/or PRMT5. HEK293T cells were transfected with pCMV-HADR4 and/or pCMV-Flag-PRMT5, respectively. The cells were lysed with lysis buffer and the lysates were then subjected to ELISA for CCL20 expression detection. $* P<0.05$.

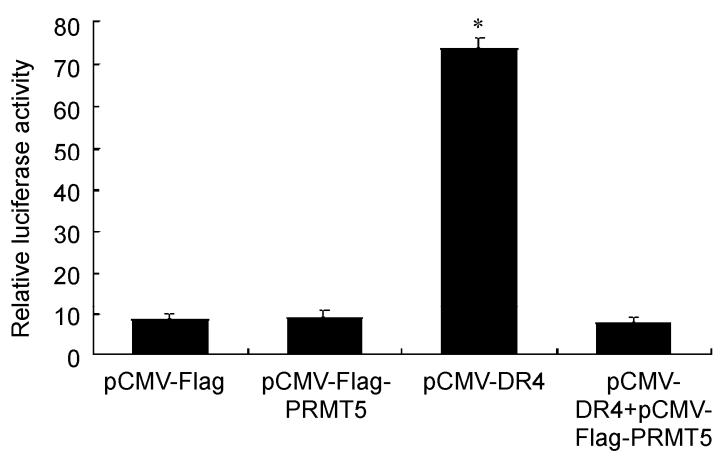

Figure 4 NF- $\kappa$ B activity in HEK293T cells with DR4 and/or PRMT5 over-expression. NF- $\kappa \mathrm{B}$ activity was depicted by the relative luciferase activity detected by using Dual-luciferase reporter gene system. $* P<0.05$.

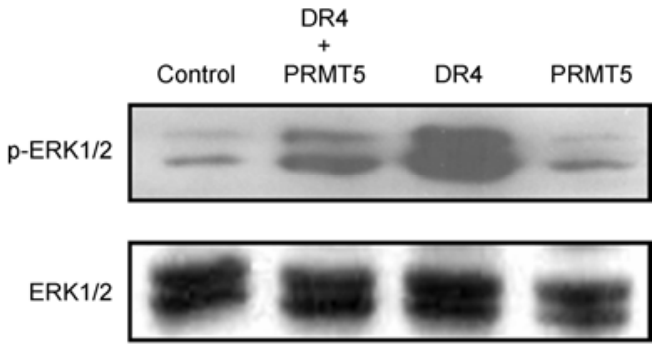

Figure 5 ERK1/2 phosphorylation in HEK293T cells with DR4 and/or PRMT5 over-expression detected by Western blot assay.

Taken together, these data demonstrate that PRMT5 suppresses CCL20 secretion via inhibiting ERK1/2 phosphorylation in NF- $\mathrm{BB}$ pathway, suggesting that PRMT5 might be involved in DR4-dependent immune regulation.

\section{Discussion}

In recent ten years, TRAIL research has been focused on its role in inducing tumor cell apoptosis and cancer therapy. However, little is known about its physiological function and the molecular mechanism. Our previous study reported that TRAIL could activate NF- $\kappa \mathrm{B}$ pathway and induce inflammatory cytokine storm, especially, an important chemokine CCL20 expression [12]. It is reported that PRMT5 interacted with DR4 in Hela cells and, therefore, interfered the activity of NF- $\mathrm{KB}$ [7], suggesting that PRMT5 might be recruited into death inducing signal complex (DISC) and further regulate cytokine release.

CCL20 is a member of chemokine family and is related to the severity of pneumonia and chronic obstructive pulmonary disease (COPD) [13]. CCL20 is highly expressed in colon cancer and scleroderma [14,15]. CCL20 could induce immune cell chemotaxis and tumor cell migration by interacting with its receptor CCR6 [16]. TRAIL plays an important role in these disease models. Up-regulated TRAIL or recombinant TRAIL combined with chemotherapy could improve the therapeutic efficacy in these diseases. These data suggest that TRAIL may have a physiological function against tumors besides inducing apoptosis.

In the present study, we have demonstrated that PRMT5 interacts with DR4, suggesting that PRMT5 may regulate the function of DISC in DR4-mediated signaling pathway. In $\mathrm{TNF} \alpha$-stimulated cells, RIP1 and TRAF2 would be recruited in the DISC and activated NF- $\kappa$ B. TRAIL, the member of the TNF super-family, may have the similar function to TNF $\alpha$. While DR4 binds with TRAIL, it could recruit RIP1 in its downstream DISC complex. TRAF2 may be also recruited in the DISC and activates NF- $\kappa$ B pathway. PRMT5 may competitively bind with DR4, and therefore, suppresses NF- $\kappa \mathrm{B}$ activation. We also observed that DR4 over-expression activated NF- $\kappa \mathrm{B}$, but DR4 plus PRMT5 over-expression suppressed NF- $\mathrm{B}$ activation, and CCL20 
expression was decreased accordingly. It suggests another possibility that PRMT5 might suppress NF- $\mathrm{BB}$ activity directly, which needs to be further clarified.

We have showed that ERK1/2 phosphorylation could be up-regulated in DR4 over-expressed cells, but suppressed in DR4 and PRMT5 over-expressed cells, indicating that MAPK/P38 pathway is involved in TRAIL-induced chemokine release. In the fact, many reseachers have reported that MAPK pathway widely participates in inflammatory cytokine release [17], which includes the cytokines presented in Figure 1, i.e., CCL20, MIP-1 $\alpha$, MIP-2B, IL-8, and TNF $\alpha$, indicating that TRAIL not only induces apoptosis, but also regulates cytokine release.

CCR6, the receptor of CCL20, is highly expressed in many immune cells, such as lymphocyte and macrophage. CCL20-CCR6 axis plays an important role in immune cell chemotaxis and migration. TRAIL or DR4 over-expression up-regulated CCL20 release, suggesting that CCL20-CCR6 interaction may involve in tumor-related immune cell infiltration or chemotaxis. The function and molecular mechanism of these immune cells in tumor growth need to be further clarified. This observation that TRAIL regulates CCL20 expression opens a novel direction on TRAIL physiological function research.

This work was supported by the National Natural Science Foundation of China (81001315 and 30972684) and the National Basic Research Program of China (2007CB507404).

1 Wiley S R, Schooley K, Smolak P J, et al. Identification and characterization of a new member of the TNF family that induces apoptosis. Immunity, 1995, 3: 673-682

2 Walczak H, Miller R E, Ariail K, et al. Tumoricidal activity of tumor necrosis factor-related apoptosis-inducing ligand in vivo. Nat Med, 1999, 5: 157-163

3 Ashkenazi A, Pai R C, Fong S, et al. Safety and antitumor activity of recombinant soluble Apo2 ligand. J Clin Invest, 1999, 104: 155-162

4 Park S J, Bijangi-Vishehsaraei K, Safa A R. Selective TRAILtriggered apoptosis due to overexpression of TRAIL death receptor 5 (DR5) in p-glycoprotein-bearing multidrug resistant CEM/VBL1000 human leukemia cells. Int J Biochem Mol Biol, 2010, 1: 90-100

5 Park S, Zhao D, Hatanpaa K J, et al. RIP1 activates PI3K-Akt via a dual mechanism involving NF-kappaB-mediated inhibition of the mTOR-S6K-IRS1 negative feedback loop and down-regulation of PTEN. Cancer Res, 2009, 69: 4107-4111

6 Mowen K A, Schurter B T, Fathman J W, et al. Arginine methylation of NIP45 modulates cytokine gene expression in effector T lymphocytes. Mol Cell, 2004, 15: 559-571

7 Tanaka H, Hoshikawa Y, Oh-Hara T, et al. PRMT5, a novel TRAIL receptor-binding protein, inhibits TRAIL-induced apoptosis via nuclear factor- $\kappa \mathrm{B}$ activation. Mol Cancer Res, 2009, 7: 557-569

8 Trauzold A, Wermann H, Arlt A, et al. CD95 and TRAIL receptormediated activation of protein kinase $\mathrm{C}$ and NF-kappaB contributes to apoptosis resistance in ductal pancreatic adenocarcinoma cells. Oncogene, 2001, 20: 4258-4269

9 Secchiero P, Gonelli A, Carnevale E, et al. TRAIL promotes the survival and proliferation of primary human vascular endothelial cells by activating the AKT and ERK pathways. Circulation, 2003, 107: 2250-2256

10 Tang W H, Wang W M, Zhang Y X, et al. Tumour necrosis factorrelated apoptosis-inducing ligand (TRAIL)-induced chemokine release in both TRAIL-resistant and TRAIL-sensitive cells via nuclear factor kappa B. FEBS J, 2009, 276: 581-593

11 Tang W H, Wang W M, Zhang Y X, et al. TRAIL receptor mediates inflammatory cytokine release in an NF- $\mathrm{BB}$-dependent manner. Cell Res, 2009, 19: 758-767

12 Wei W, Wang D S, Shi J, et al. Tumor necrosis factor (TNF)-related apoptosis-inducing ligand (TRAIL) induces chemotactic migration of monocytes via a death receptor 4-mediated RhoGTPase pathway. Mol Immunol, 2010, 47: 2475-2484

13 Demedts I K, Bracke K R, Pottelberge G V, et al. Accumulation of dendritic cells and increased CCL20 levels in the airways of patients with chronic obstructive pulmonary disease. Am J Respir Crit Care Med, 2007, 175: 998-1005

14 Tao J, Li L, Tan Z, et al. Up-regulation of CC chemokine ligand 20 and its receptor CCR6 in the lesional skin of early systemic sclerosis. Eur J Dermatol, 2011, 21: 731-736

15 Liu J L, Zhang N, Li Q, et al. Tumor-associated macrophages recruit CCR6+ regulatory T cells and promote the development of colorectal cancer via enhancing CCL20 production in mice. PLoS One, 2011, 29: e19495

16 Rubie C, Frick V O, Ghadjar P, et al. Effect of preoperative FOLFOX chemotherapy on CCL20/CCR6 expression in colorectal liver metastasis. World J Gastroenterol, 2011, 26: 3109-3116

17 Johnstone R W, Frew A J, Smyth M J. The TRAIL apoptotic pathway in cancer onset, progression and therapy. Nat Rev Cancer, 2008, 8: $782-798$

Open Access This article is distributed under the terms of the Creative Commons Attribution License which permits any use, distribution, and reproduction in any medium, provided the original author(s) and source are credited. 